\title{
LA VALEUR POUR UNE SÉMIOTIQUE DES PRATIQUES
}

\section{VALUE FOR ONE SEMIOTICS OF PRACTICES}

PIERLUIGI BASSO FOSSALI *

RÉSUMÉ : Même si on peut analyser en manière légitime la valeur comme une couverture lexicale qui cache des élaborations conceptuelles très hétérogènes, l'article cherche d'en trouver un socle commun et des axes de caractérisation. $\mathrm{Au}$-delà de la fondamentale élaboration saussurienne, la valeur semble avoir une thématisation privilégiée en économie et en éthique ; toutefois, aujourd'hui elle est au centre des stratégies de marques et les intangibles ont construit un véritable marché identitaire qui semble soumettre l'économie aux valeurs élaborées dans les échanges communicatifs. Enfin, le tournant « expérientiel » du marketing semble inviter à saisir les conversions entre des valeurs discursives et des valeurs perceptives. Ces effervescences sociales peuvent pousser la sémiotique à réveiller la réflexion théorique sur la valeur, en cherchant un nouvel potentiel explicatif et critique de ses modèles. L'exploration conduite dans cet article cherche de combler quelques vides théoriques qui séparent les thématisations phénoménologiques, linguistiques et sociologiques de la valeur; en particulier, nos objectifs

* Université Lumière Lyon 2 - Laboratoire ICAR, ENS de Lyon. E-mail: pierluigibasso@ hotmail.com. 
principaux sont une nouvelle typologie des valorisations et un éclaircissement des aspects constitutifs de la valeur mis en tension par la dialectique entre les programmes narratifs et les réserves figuratives des acteurs.

MOTS-CLÉS : Valeur. Perception. Engagement. Implication. Figurativité. Narrativité.

ABSTRACT : Although the value as a lexical coverage that hides very heterogeneous conceptual elaborations may be legitimately analyzed, the article seeks to find a common ground and characterization axes. Beyond the fundamental Saussurian development, the value appears to have a unique thematization in economics and ethics; however, it is nowadays the focus of brand strategies. Besides, intangibles have built a true identity mark that seems to submit economy to the values developed in communicative exchanges. Finally, "experiential" marketing environment seems to prompt to leave conversions between discursive values and perceptual values. This social effervescence can lead semiotics to reveal theoretical reflection on the value in seeking a new explanatory and critical potential of its models. The exploration conducted in this paper seeks to fill some theoretical gaps that separate phenomenological, linguistic and sociological thematizations of the value; in particular, our main goals are a new typology of valuations and a clarification of the constituent aspects of the value put in tension by the dialectic between narrative programs and actants' figurative reserves.

KEYWORDS : Value. Perception. Commitment. Involvement. Figurativity. Narrativity. 


\section{Valeur et valence}

\section{Prééminence de la parole : valeur d'usage et distance axiologique}

Nous voudrions commencer ex abrupto par une définition provisoire : la valeur établit le caractère opératif du signifié dans un espace (discursif) de référence mobilisé en fonction d'une détermination identitaire (d'ordre différentiel). Le privilège accordé au terme valeur est le corrélat du primat de la parole, donc de l'énonciation en acte, par rapport à la signification en langue. La valeur dégénéralise la signification et procède d'un programme de caractérisation des identités culturelles. Il faut donc reconsidérer la valeur en la situant dans le niveau de pertinence des pratiques; si l'on a parlé des valeurs purement différentielles, c'est parce qu'elles ont été considérées avant tout dans les systèmes ou dans les textes, c'est-à-dire avec l'avantage d'une clôture et d'une autonomie de l'espace de référence. La clôture permet le calcul et concède l'attribution de sèmes ou de rôles à une entité sémiotique, car tels sèmes ou tels rôles ne sont pas réclamés par d'autres entités co-occurrentes (SAUSSURE, 2002, p. 81). La codification peut être vue comme la préservation en captivité des valeurs qui, dans l'expérience, ne peuvent trouver ni une commensurabilité (chaque lien implicatif est un appel individualisé à l'existence), ni une accumulation (la valeur en acte est comme l'électricité, elle subsiste dans un flux de présence). Une telle captivité semble être la médiation nécessaire pour régler l'asymétrie sociale et temporelle des ressources ${ }^{1}$ et pour réduire l'indétermination

1 Le patrimoine sémantique des codes a toujours un potentiel consolatoire par rapport à la distribution inégale des valeurs et des chances. 
des communications. Toutefois, si

[...] le signifié d'un mot (plus précisément : d'une lexie) est défini comme une valeur, les différences qui constituent cette valeur définissent le contenu opératoire de la lexie, c'est-àdire l'ensemble de ses possibilités de combinaison dans les textes. Chaque trait est déterminé par des récurrences contextuelles, d'où l'impression qu'il est pourvu d'une valence (RASTIER, 2011, p. 30, c'est l'auteur qui souligne).

Il y a une mémoire opérative par rapport à un corpus d'usages (valences doxiques), aussi bien qu'une disponibilité à l'investissement dans d'autres cotextes (espaces discursifs de cooccurrences). Enfin, on pourrait aussi saisir la valence d'une valeur dans les passages entre des niveaux de pertinence (signes, textes, objets, pratiques, etc.) :

L'intégration au niveau supérieur étant la condition pour que l'unité trouve sa valeur distinctive au niveau inférieur, nous retrouvons ici, avec d'autres termes, le différentiel des modes d'existence, puisque la valeur distinctive reste « potentielle » tant que l'unité n'est pas intégrée au niveau supérieur : c'est l'intégration qui la "réalise" (FONTANILLE, 2008, p. 45).

Toutefois, le niveau de pratiques prévoit un régime particulier de valences pertinentielles ; en effet, chaque pratique sémiotique est une gestion du sens ouverte qui construit localement et progressivement son espace de pertinence en terme de dialectique entre des valeurs opératives (hétéronomes et contraignantes) et des valeurs opérables (disponibles à la prise d'initiative) (BASSO FOSSALI, 2006; 2009a). La valeur différentielle devient alors la valeur expérientielle ou discursive qui fait la différence. Il y a deux conséquences immédiates de cette prise de position 
théorique :

(i) la première est le désaveu du parallélisme entre la valeur en sémiotique et la valeur d'échange dans les sciences économiques, étant donné que la seconde pourrait éventuellement analogiser les signifiés linguistiques dans les relations paradigmatiques d'équivalence, mais alors elle cacherait inévitablement le « valoir » de telles valeurs, c'està-dire leur valence implicative (faire la différence). La vision économique construit un dispositif de commensurabilité, voire de traductibilité, de toutes les valeurs à travers une virtualisation des valences : le travail humain, l'argent, les biens matériaux, les intangibles sont tous équivalents dans la perspective de l'échange, qui laisse par la suite aux acteurs sociaux le calcul de l'intérêt personnel (la monnaie devient la traductrice universelle, qui « lave » les traces de la production et dispose l'intraçabilité des dépenses²). En revanche, la valeur en sémiotique ne peut pas être détachée de l'acte d'appréhension perceptive ou de l'énonciation, en mettant en jeu nécessairement, selon des assomptions ou des « rejections », une implication dans la relation individuelle ${ }^{3}$. La valeur linguistique peut revendiquer plutôt une connexion théorique avec la valeur d'usage.C'est pourquoila comparaison

2 On pourrait argumenter que chaque valeur exprime une intensité d'implication qui est appréciable seulement si on la met en relation avec celle d'autres valeurs. Même la valence relèverait alors d'un système capable de fournir des paramètres abstraits de confrontation. Or, le prix économique pourrait exemplifier l'obstacle à l'expression du désir, le « quantum de résistance qui peut être rencontré devant tout objet valorisé» (CLAM, 2006, p. 57). Cependant, cette exemplification abstraite est limitée à l'intensité de la valeur et surtout elle est, par définition, spécifique par rapport aux enjeux identitaires et aux espaces de référence.

3 Même la distinction entre les valeurs différentielles et les valeurs phénoménologiques n'était alors qu'un effet de la subordination de la parole à la langue et de la scénarisation communicative (ancrage figuratif) à la signification abstraite (sémio-narrative ou logique). 
entre langue et argent doit être explorée par rapport à deux aspects partagés : d'une part, le caractère intangible des valeurs traitées (RASTIER, 2004) et, de l'autre, la corrélation, saisie selon une perspective intersémantique ${ }^{4}$, entre des valences hétérogènes et douées chacune d'une fluctuation distincte (par exemple, appréciations/dévaluations des monnaies nationales et des marchandises).

(ii) la seconde conséquence est que la valeur est saisie dans des faisceaux de relations implicatives bilatérales (sujet/ objet ou ego/alter) et qu'elle enregistre donc toujours une distance axiologique, qu'elle soit implicite ou explicite. Le global (axiologie) engage le local (valeur) tout comme la méréologie identitaire hypothèque le traitement de traits figuratifs. C'est ce qui permet à la sémiotique générale de saisir la question communicative (c'est-à-dire la renégociation de l'asymétrie des valorisations) comme déjà enracinée dans la conception même des valeurs. La valeur n'émerge que dans la relation intersubjective, étant donné que le lien implicatif qu'elle exprime va décider d'une définition d'un trait de soi par rapport à un trait d'autrui (par exemple, en termes d'intercorporalités ) selon des polémiques ou des solidarités distinctives. Toutefois, il faut ajouter que la valeur exprime les fractures internes à la doxa et souligne ainsi l'afférence à des appréciations qui valent pour leur distanciation réciproque.

\section{Approfondissement de la valeur et sublimation}

Un lien implicatif est déjà en soi une inégalité

\footnotetext{
4 Cf. § « Détermination positive de la valeur et plan de l'expression ».

5 Nous faisons allusion évidemment à la leçon de Merleau-Ponty.
} 
qualitative qui déséquilibre l'élaboration du sens vers un enjeu identitaire particulier (focalisation attentionnelle) et une résolution figurative du scénario expérientiel (écologie sélective ${ }^{6}$. Toutefois, les confrontations interactancielles vont s'accumuler, de sorte qu'elles doivent être hiérarchisées et localement neutralisées au profit d'autres fronts émergents. Cela explique de nouveau le recours à la notion de valence, c'est-à-dire « le valoir » de la valeur : la valence est alors la prééminence locale d'un lien implicatif par rapport à d'autres valeurs en acte sur le plan de la confrontation interactantielle. Sans des valences, les valeurs resteraient équivalentes et donc dans un état de neutralisation réciproque qui ne suffirait jamais à donner une consistance sémantique et une dynamique aux scénarisations. En outre, la valence réduit l'indétermination modale des valeurs en s'offrant comme un moteur d'interprétation ${ }^{7}$, comme une mobilisation orientée d'interprétants. La valence donne en effet une définition minimale de l'accès au sens qui est toutefois beaucoup plus développée et dense que la vieille opposition euphorie/ dysphorie, manifestement surestimée par la sémiotique greimassienne. Les valences sont la mémoire de l'ouverture originaire des scénarisations qui affecte même les systèmes de valeurs plus codés ; mais elles sont aussi le symptôme de l'intériorisation défective des rôles sociaux négociés.

Le circuit sémiotique des valences informe les procès de socialisation des rôles tout comme leur psychologisation, en garantissant une gestion du gradient d'implication, voire une

6 On peut retrouver ici un parallélisme avec la sémiotique tensive, étant donné qu'elle conçoit la valeur comme le quotient de l'intersection entre une visée et une saisie. Dans notre argumentation, il y a aussi de prise de distance par rapport à ce tournant de la sémiotique post-greimassienne.

7 Cf. § «Différences et valences : la valeur même de la sémiotisation ». 
sublimation des implications. En effet, même des valeurs qui semblent plus résistantes au devenir ou plus indispensables (attachement) peuvent être sublimées. Normalement, la substitution par « équi-valence » des termes échangés prévoit une traduction économique, avec des valences hétéronomes qui construisent une certaine commensurabilité (monétisation et disponibilité) ; en revanche, la sublimation est une transposition dans un autre domaine, qui obtient une valence comparable a posteriori. La redirection des thématisations parie sur une restructuration identitaire (création) capable de préserver, voire améliorer, le sentiment de validité des valeurs qu'on peut investir à l'extérieur ou s'auto-attribuer. La sublimation motive la transversalité du sens de la rhétorique (figuralité), même sans en garantir l'efficacité : de fait, pratiquer la rhétorique consiste à « jouer » à la sublimation, même après l'avoir parfois transformée en pur jeu.

\section{Les valeurs : entre expérience et discours}

\section{Différences et valences : la valeur même de la sémiotisation}

Si on doit se méfier de la pure différence, il serait imprudent de déprécier le rôle de la différence dans l'organisation des valeurs. La centralité théorique de l'implication dans la gestion sémantique nous renvoie, en amont du terrain linguistique, vers une sémiotique de premier ordre, ancrée à l'expérience perceptive ; ici, la valeur est immanente à un espace d'enracinement implicatif univoque (inhérence) et elle est immédiatement reconduite à des enjeux d'individuation, en particulier à l'établissement perceptif d'un cadre de relations interactantielles (BASSO FOSSALI , 2009b). C'est pourquoi la valeur est éminemment 
relationnelle et différentielle, c'est-à-dire écologiquement orientée vers l'assimilation/dissimilation identitaire. La sémiotique discursive profite en revanche de la logique des hiatus imaginatifs pour détacher les valeurs des enjeux implicatifs ancrés sur le plan perceptif et les projeter sur un espace fictif, au bénéfice d'une élaboration différentielle capable d'organiser de nouveaux profils identitaires (simulacres discursifs).

L'élaboration culturelle n'est que la multiplication de modélisations discursives qui nous permettent de sortir des descriptions précédentes; la " santé » des acteurs sociaux se préserve dans leur capacité à ne pas rester prisonniers des jeux de langage, en multipliant les implications dans des terrains différemment réglés. On cherche la différence. Nous nous en permettons une explicitation ultérieure, selon un cadre théorique d'inspiration peircienne, où l'on trouve des valences impliquées dans les catégories les plus générales de la sémiotisation (priméité, secondéité, tiercéité) (BASSO FOSSALI , 2011):

(i) les valences «médiatives » (tiérciété) soulignent le poids de la réargumentation dans la lutte contre la désémantisation qu'affecte toute modélisation discursive ;

(ii) les valences « éxistentives » (secondéité) affichent en revanche la nécessité des principes régulateurs dans les assomptions différenciées des valeurs par rapport à l'horizon destinal ;

(iii) les valences diagrammatiques (priméité) nous accordent la possibilité de projeter des formes de relation entre des champs thématiques divers. 
Ces trois types de valences ne sont que les axes de sémiotisation récursive des valeurs qui profilent leur exploitation dans des directions interprétatives. En effet, les valeurs ne fixent pas seulement les points de repérage des scénarisations, mais elles s'offrent aussi comme les ancrages des analogisations (priméité), des enquêtes indiciaires (secondéité), des nouvelles pertinentisations (tiercéité).

\section{Valences perceptuelles et valences discursives}

L'expérience, dès qu'elle est culturalisée avec une sémiotique de second ordre, doit gérer une coalescence des valeurs élaborées perceptivement et des valeurs qui sont construites et négociées à travers les architectures discursives ; une telle coalescence peut viser à l'homogénéisation des valences ou à leur compartimentation. Du reste, les mouvements interprétatifs qui cherchent à reconstruire un fil commun entre l'hétérogénéité de telles valences ne reproduisent que les fluctuations parallèles des valeurs en langue et des valeurs en expérience, voire ils construisent des évaluations paradigmatiques des entités discursives et des entités perçues.

La recherche d'une homogénéisation est typique de la forme de vie qui tôt ou tard cherche une transpiration expérientielle des valeurs identitaires négociées dans la vie sociale ; en revanche, la compartimentation dépend des domaines sociaux qui visent à garantir une administration protégée des valences spécifiques, même dans l'évidence d'un manque de fondement (valences juridiques pour le droit, valences esthétiques pour l'art, etc.). Il y a une tension constante entre des instances d'homogénéisation et des 
instances de compartimentation : d'une part, l'immersion perceptive oblige à une gestion des valeurs imperfectives, toujours ouverte spatialement et temporellement ; de l'autre, au niveau discursif, il y a toujours la tentation de fixer des clôtures narratives, des termes contractuels, des conclusions argumentatives. Les vécus de signification ne sont que les pulsations interprétatives obligées de relier les valences perceptuelles avec les valences discursives selon une résolution de l'hétérogénéité qui est finalement narrative et qui conserve les déterminations et les conclusions comme une illusion nécessaire. Malgré les caprices de la fortune, la valence se pose toujours dans les réouvertures destinales, mais il faut remarquer que la fin scelle des couronnements et des congés ont un potentiel réinterprétatif sans égal.

\section{Clôtures narratives et imperfection du sens}

Les discontinuités existentielles sont des valences discursives qui trouvent leur plus large attestation dans les cultures, car elles donnent à voir une détermination des valeurs, qui souffrent en revanche, sur le plan de l'expérience perceptive, de l'incapacité chronique de parvenir à leur pleine réalisation (imperfection). L'implication corporelle de l'émotion témoigne toujours du procès imperfectif de l'expérience et de l'ouverture constitutive (quotient d'indétermination) de chaque espace pratique. L'élaboration des rôles passionnels aspire en revanche à idéaliser une pointe de dissolution (tragique ou glorifiante) des enjeux existentiels, une preuve décisive pour le perfectionnement d'une élaboration narrative de l'identité. La narrativité est alors une synthèse de l'hétérogénéité expérientielle (valences 
perceptives et discursives) qui doit expier ses défaillances constitutives dans la "réalisation » du soi à cause de l'intromission du continuum imperfectif de l'émotion.

\section{Concurrence des valeurs}

On sait que la théorie de la valeur chez Saussure tire son inspiration de la tradition anglaise des études d'économie; or, une réflexion intéressante qu'on peut faire dans ce rapport interdisciplinaire est que le fait de combler un manque ne peut pas nous dédommager d'autres lacunes existentielles. La prééminence d'une relation visée est conjoncturelle et ne résout pas la concurrence subjacente d'autres valeurs, d'autres manques. La forme de vie est atteinte d'un «plurilatéralisme » congénital.

On peut bien soutenir qu'une valeur n'est jamais dépourvue en soi-même d'une tension relationnelle engendrée par des programmes de recherche ou de maintenance, mais sa valence reste tout autre chose, à savoir une inflexion particulière et compétitive qui affecte un scénario d'implication, certainement doué d'une complexité beaucoup plus vaste ${ }^{8}$. En effet, le déséquilibre que produit la valence, par rapport au réseau composé par d'autres valeurs en acte, n'est pas superposable au choix des points d'intervention auxquels la prise d'initiative se confie. Au contraire, la valence locale « harcèle » souvent les protocoles d'action qui ont déjà établi des paramètres de rationalité. Dans cette perspective, les valences expérientielles ont un pouvoir de contestation

8 «La valeur est comme l'état refroidi du désir dans sa distance par rapport à sa satisfaction ou dans l'éloignement des objets de sa satisfaction. Elle mesure cette distance même comme une distance vécue, soutenue, intégrée de manière fondamentalement non problématique à la forme de vie» (CLAM, 2006, p. 44). 
sur les valeurs qui suivent le " fil du discours », étant donné qu'elles montrent des valeurs compétitives en vigueur et avec une implication incontestable (inhérence du corps à l'environnement). Parallèlement, il faut admettre que les avantages de l'expérience dans l'exemplification des valences rendent encore plus « héroïque » et élaborée que l'affirmation de valences établies sur le plan discursif. Il faut proportionner les exigences d'homogénéisation cognitive avec les appels constants des implications expérientielles, qui empêchent dans tous les cas de résoudre l'environnement dans une reproduction représentationnelle : l'ancrage de valences expérientielles reste le couplage avec l'entour.

\section{Détermination positive de la valeur et plan de l'expression}

Dans une signification « à plein régime », il n’y a pas constitution de valeurs pures ou « aurorales»; elles dépendent d'un horizon d'attente et de scénarisations prototypiques (frames) qui dénoncent une écologie figurative (recherche d'une consistance sémantique) et le respect d'un paradigme configurationnel (enquêtes identitaires ouvertes). En effet, chaque valeur relève d'une configuration et donne donc une détermination positive à cette dernière, en termes d'accomplissement relatif ou de recomposition d'une méréologie identitaire. Naturellement, la valeur reçoit aussi en retour une détermination positive.

Or, la fin d'un texte linéaire (par exemple, un roman) sur le plan de l'expression a été prise comme l'indice d'accomplissement sur le plan du contenu (fermeture des relations intratextuelles) qui permet une relecture de toute la configuration comme un enchainement de présuppositions. Nous avons déjà remarqué que la clôture a servi de prétexte 
pour transformer même le plan du contenu dans un paysage de relations purement différentielles selon des attributions distribuées de traits et de rôles jusqu'à la saturation. Cela protégeait, par exemple, la sémiotique greimassienne d'une irruption ingérable de valeurs phénoménologiques, infléchies par des valences qui suivent les caprices du devenir (extéroceptif et intéroceptif), même s'il fallait pour cela laisser la théorie narrative dans un état d'ambiguïté ${ }^{\text {: valeur }}$ différentielle ou valeur considérée comme « sens de la vie $»^{10}$ ?

La reconnaissance de la problématique du «valoir» de la valeur aurait obligé à explorer une détermination bien plus avancée du caractère " positif » de la valeur ; cette détermination restait en revanche l'effet patent d'un accomplissement méréologique par des assignations de traits différentiels jusqu'à la saturation des dissimilations/ assimilations interne au texte. Au fond, la peur même d'une réduction des médiations sémiotiques au rôle d'une monnaie d'échange se transformait par réaction dans le refoulement de l'intersémantique et de la gestion pratique de la signification, obligée de respecter l'ouverture indéfinie de l'expérience (toutefois, c'est l'expérience qui oblige à baliser les assomptions de valeurs, en mettant à l'épreuve les mondes

9 La sémiotique des passions a cherché à résoudre cette ambiguïté mais à travers un palier de préconditions de la signification qui finalement était prêt à reconnaitre l'ouverture de la signification comme un terminus ab quo et non comme un terminus ad quem.

10 «Pour Greimas il s'agit, semble-t-il, de l'identité entre d'un côté l'impossibilité de saisir le sens des signes autrement que par son articulation en valeurs linguistiques et d'un autre côté l'impossibilité de saisir imaginairement le "sens de la vie" autrement que par l'enchaînement d'actions et de projets dont la valeur existentielle n'apparaîtra qu'après-coup, lorsque la sanction dernière aura transformé notre existence en destin.» (PETITOT, 1985, p. 291). Greimas a répondu implicitement à une telle lacération théorique avec De l'imperfection (1986); le débat s'organise autour de la question de savoir si cette lacération a été suturée ou accrue encore plus pour envisager un tournant de la sémiotique. 
discursifs trop librement élaborés). Pour ce qui concerne l'intersémantique, elle ne reproduit pas la question de la référence, mais elle ouvre plutôt un champ de connexions et d'appréciations entre corpora ou domaines construits par des sémiotisations différentes ; toutefois, il faut reconnaître la spécificité des valences expérientielles, étant donné qu'elles sont le paramètre même de l' «être dans le langage », à travers l'exemplification de l'inhérence du corps à l'environnement.

Cela conduit à reconnaître que la valeur codirige l'orientation des transformations et que sa détermination ne peut pas être seulement "négative ». Sans recouper un paradigme "réaliste », la sémiotique peut s'inscrire dans une perspective épistémologique " constitutionnaliste » qui défende la double dépendance (subjectale et objectale) des valeurs qui s'inscrivent dans le couplage entre un épicentre de valorisations et un environnement largement sémiotisé. Il n'y a donc pas d'élaboration des valeurs qui précède les questions dirimantes d'auto-attributions et d'imputation des traits. La seule chose qui oppose la perception à l'énonciation est le moment de l'assomption : dans l'expérience, tout commence avec une question d'embrayage (l'environnement est une implication irrémissible) ; dans le discours il semble essentiel avant tout de procéder à la déclinaison d'un monde figuratif (débrayage d'un espace fictif), même si la stratégie énonciative n'est, dès le début, que la tentative d'activer des imputations et des auto-attributions. Différence (assimilation/dissimilation) et détermination positive des valeurs sont alors les deux faces de la même question, si l'on accepte que les relations se constituent selon des paramètres cosystématiques (la double dépendance des déterminations est aussi une double afférence, un croisement de formations identitaires). 
Même la valeur sur le plan de l'expression n'est que le produit « positif » de la sémiotique discursive qui va étendre le champ du "significatif » à la gestion des corrélations linguistiques ; c'est-à-dire, les langages produisent des hiatus de signifiance entre des valeurs - construites par deux pertinences différentes (formes) - afin de leur faire interpréter l'un le rôle de signifiant, l'autrele rôle de signifié. Les signifiants sont aussi des biens culturaux qui ont une identité par rapport à la langue et qui, pour cette raison, deviennent toujours, en dernier ressort, des interprétants des passages textuels où ils sont utilisés (RASTIER, 2001). Le gradient d'abstraction dans l'élaboration de l'expression (prélèvement sélectif et projection articulatoire des diagrammes) ne dénonce que le travail de l'élaboration culturelle et son ouverture à la renégociation.

\section{Économies des valeurs}

\section{Valeurs à consommer et exigences cosystématiques}

Si on doit reconnaître que chaque valeur répond à une exigence cosystématique, il faudrait aussi souligner que l'échange peut bien se limiter à une simple substitution paradigmatique mais souvent il finit par marquer un passage d'un système à un autre ; si dans le premier cas on trouve immédiatement une garantie de commensurabilité, dans le second il faut expérimenter un nouvel équilibre, étant donné que la distribution des valences entre les divers profils identitaires n'est plus assurée. La gestion des valeurs passe par des administrations identitaires (conservation, innovation, etc.), des formes de sélection (élection, accumulation, etc.), des stratégies de collectivisation ou de privatisation qui 
démontrent toutes l'ancrage intersubjectif des traitements ${ }^{11}$.

Dans les pratiques de consommation, les valeurs identitaires, avec leur côté figuratif, sont les pivots commerciaux qui permettent la substitution périodique de produits améliorés et la gestion des valences de leur classe d'appartenance. On opère sur les caractères matériaux aussi bien que sur les rôles idem et ipse des produits pour négocier ou cacher des succédanés, des marchandises certifiées, des ruptures de classements commerciaux habituels. Les échanges intra-paradigmatiques sont alors possibles aussi bien que les échanges interparadigmatiques, dans lesquels la comparaison sémantique s'actualise en termes d'expérimentation des nouveaux équilibres (restructuration des pratiques et des habitudes ; et pourtant, on ne trouvera jamais la promotion des formes de vie anti-systématiques, comme par exemple la pauvreté, capable de construire, à partir des manques perçus, une nouvelle dévotion au monde. La monétisation deséchanges permet plutôt des équivalences inter-paradigmatiques qui laissent fondamentalement inaltérées les vocations identitaires, même à l'intérieur d'un circuit assuré des rôles sociaux permutables (producteur, consommateur, financier). À ce propos, la marque impose un « logiciel » identitaire qui est approprié au profil du producteur, du consommateur et même du produit de consommation. L'égalisation idéologique, qui cache les asymétries des intérêts et des statuts et qui oblige à un surplus de communication, montre perversement que les exigences cosystématiques dans la production de valeur sont perçues et respectées par le marché.

11 Nous manquons d'espace pour analyser ces traitements de valeurs et nous nous permettons par conséquent de renvoyer à notre monographie sur cet argument, dans Basso Fossali (2008a). 


\section{Valeur ajoutée}

Par rapport à nos argumentations initiales ${ }^{12}$, il faudrait reconnaître que, dans l'économie classique, le concept de valeur est compliqué par l'idée qu'il exprime la possibilité de permuter des biens qui ont la même difficulté à être produits et/ou acquis. L'échange n'est alors que le recouvrement superficiel et terminatif d'une scène pratique où toute l'évaluation des valeurs des biens échangés se situe en amont du mesurage monétaire. La substitution de propriété des biens est réglée dans tous les cas par des appréciations ou des dévaluations indépendantes d'autres formes d'implication que la production et l'acquisition : cela n'est que l'affirmation d'un paradigme financier, où la détermination des investissements substitue l'indétermination des gains identitaires et des satisfactions. Les valeurs économiques offrent alors des mobiles rationnels pour les investissements, qui permettent dans le même temps d'éloigner vers l'horizon destinal le plus incertain une véritable évaluation des implications et donc des valences hétéronomes : l'accumulation de biens ou d'argent se justifie par la quantité d'incertitude externe au paradigme financier ou, mieux, on préfère des incertitudes internes (l'économie est aussi une économie d'incertitudes).

Il faut également prendre en considération l'évaluation récursive de l'investissement (valence du prix par rapport à des financements ultérieurs) ; c'est pourquoi l'on est toujours en train de créer de la valeur ajoutée et que cette création se développe surtout sur le plan de la communication. La négociation des traits identitaires dans les espaces discursifs permet de passer de la détermination située et concrète à

12 Cf. § «Prééminence de la parole : valeur d'usage et distance axiologique ». 
un constructivisme incrémental de nature idéale ; en effet, la valeur ajoutée n'est pas seulement la mesure qui quantifie la différence entre les coûts de production (consommation intermédiaire) et les gains obtenus par la collocation de produits sur le marché ; les intangibles démontrent l'existence de valeurs ajoutées obtenues seulement à travers des attributions performatives et des associations discursives : changement de marque des produits, restyling du logo, etc.).

La valeur ajoutée ne se limite pas à enregistrer le bénéfice net par rapport aux dépenses ; la redirection des valeurs dans de nouveaux champs d'investissement est certes un moyen de continuer à produire de la valeur (par exemple, en termes de brand extension), mais il faut remarquer que l'adjonction implique aussi la redéfinition des identités et pas seulement des relations d'investissement. Même les traits identitaires « adjoints » peuvent entrer dans le régime de dépense et être réinvestis. Le marché de valeurs n'est pas seulement un marché de monnaies, mais une recomposition identitaire sous une logique d'investissement et de dépenses (marché identitaire). Les identités discursives ne sont pas structurées comme des corps mais comme des réservoirs disponibles aux réorganisations ; il s'ensuit de là que les acteurs sont conçus comme des réserves identitaires toujours prêtes à la restructuration et à la réorganisation (boulimie des valeurs ajoutées), selon un vidage progressif des noyaux d'enracinement. Le culte de la personnalité n'est peut-être qu'une réaction qui affiche typiquement les paradoxes internes à la culture. 


\section{Institutionnalisation des valeurs et affectation de leur valence}

Nous ne pouvons pas oublier que les valeurs institutionnalisées répondent à une exigence de rationalité ; toutefois, même si elles sont organisées par les domaines sociaux qui en administrent les valences, elles restent toujours une motivation de souffrance. Il y a un «poids » des valeurs institutionnalisées qui concerne leur scénarisation événementielle (sujétion imprévue et captieuse) et qui contraste avec la perspective pragmatique où elles se traduisent facilement dans une sélection d'objectifs et de moyens. Du reste, l'utilitarisme doit également faire face à une intériorisation généralement défective de valeurs institutionnalisées. En effet, la mobilisation rationnelle, toujours guidée par l'offre des valences déjà socialisées par des domaines tels que l'art, la science, la religion, etc., souffre d'un décalage endémique entre l'idéalité des manques et leur échappement à la codification et à l'organisation. Pour cette raison, on ne cesse jamais de communiquer le défaut d'intériorisation des valences sociales et la nécessité d'expérimenter un " poids » des valeurs in vivo. Les circuits des valeurs institutionnalisées tendent à réclamer une « mise à terre » locale, d'ordre expérientiel, mais celle-ci construit immédiatement un clivage entre la perspective négociée et l'intériorisation effective. Le poids des valeurs est destiné à une expérimentation asymétrique par les divers sujets sociaux, même si leur institutionnalisation viserait à l'égalisation des conditions de jouissance.

Il y a toujours en jeu une double contrainte (double bind) : d'une part, on sollicite la cooptation des valeurs préorganisées ; de l'autre, on apprécie leur appropriation 
filtrée par une distance critique opportune et, possiblement, par une conversion de valences tout à fait personnelle. Finalement, on doit adhérer aux rôles identitaires élaborés dans un certain jeu de langage et dans le même temps préserver une position dans un autre jeu qui puisse exemplifier l'accès à des valeurs diversement compétitives. L’adhérence totale aux valeurs institutionnalisées serait une dévotion radicale de la subjectivité en tant qu'épicentre autonome de valorisations ; en revanche, la multiplication des implications à travers des jeux de langage divers peut produire une «faim » endémique, alimentée par la recherche d'une saturation de toute valence disponible dans les marchés sociaux. Cela explique le défaut de conséquentialité entre les fins initiales et les décisions intermédiaires ou finales : d'une part, il y a l'ambition malade de vaincre sur tous les plans de la confrontation sociale (saturation des enjeux identitaires) ; de l'autre le refus salutaire de la superposition entre une définition extrinsèque et intrinsèque de soi (objectivation et idéalisation du soi peuvent bien se contrebalancer).

On peut trouver dans l'écologie des formes de vie (BASSO FOSSALI, 2012) une dialectique constante entre les recherches locales d'une direction de vie (implication intensive) et d'un cadre d'existence (implication extensive). La « linéarisation » des prises d'initiative (parcours narratifs) est alors toujours contrastée par une appréhension « tabulaire », synoptique qui tire profit de l'hétérogénéité plurivoque des fronts d'individuation. 


\section{Les valorisations}

\section{Valorisations réflexives et transitives}

La valorisation n'est que la tentative d'ancrer les valences dans un cadre stratégique qui puisse réduire la complexité d'implications d'un espace de référence. La valorisation est donc une anticipation d'investissements orientés par rapport aux implications événementielles qui pourraient s'imposer localement ${ }^{13}$; en particulier, elle peut se pencher vers le versant objectal (valorisation transitive) ou le versant subjectal (valorisation réflexive) d'une certaine relation ${ }^{14}$. On pourra distinguer les valorisations réflexives suivantes :

a) les valorisations destinales promeuvent des valeurs qui s'offrent au sujet observateur pour une pleine assomption dans sa propre trajectoire existentielle et qui peuvent donc s'avérer décisives pour l'affirmation ou la négation de sa « tenue » identitaire dans le temps. Souvent, les valorisations destinales transforment des détails latéraux ou accidentels en symptômes d'une ruine ou en signes anticipateurs du bonheur ;

13 Les valorisations peuvent apparaître comme une nouvelle catégorisation de ce que la doxa appelle "fonctions », en les traduisant dans des signifiés relationnels (homogénéisation théorique) et en les dégénéralisant (les valorisations désignent des rapports d'assomption et des déséquilibres locaux, pas des rôles universels).

14 Cf. Basso Fossali (2008b). Ici, nous nous limiterons à résumer l'essentiel de cette étude plus vaste. Par exemple, il faudrait thématiser la possibilité d'une réflexivité et d'une transitivité des valorisations qui s'enracineraient dans les stratégies des objets ; cela nous conduirait à parler des valorisations « destinales » d'un objet et des valorisations «prestationnelles » des sujets. Toutefois, cette inversion serait problématique, car la distinction même entre les objets et les sujets relève de l'impossibilité des premiers à se proposer comme des épicentres autonomes de valorisation, bien qu'ils deviennent des relais de valorisations intersubjectives dans un réseau institutionnel d'implication. 
b) les valorisations formatives sont concentrées dans l'idée que le passage par l'expérience de l'altérité est fondamental afin d'accroître sa propre compétence et de fuir, ainsi, un destin fermé ; l'assomption des propositions hétéronomes risque toutefois la cooptation acritique de modèles jusqu'à l'émulation ;

c) les valorisations digressives, même si elles suspendent les visées fondamentales d'une forme de vie, nient les préoccupations concernant l'altérité ; on cherche des variations de vécus capables d'exprimer des aspects minoritaires de sa propre identité, sans nécessairement une compromission de la direction de vie ; toutefois, ces variations peuvent instiller des attitudes dispersives ;

d) les valorisations émancipatives nient l'obligation d'avoir un destin propre et favorisent la multiplication des points de vue sur les valeurs disponibles, exemplifiées par l'altérité (hétéronomie des sollicitations), en démontrant dans le même temps la capacité de savoir passer avec agilité d'implication en implication, d'investissement en investissement. Les valorisations émancipatives peuvent corroborer des attitudes sceptiques, au prix d'une perte presque totale d'enracinement. 
Figure 1 - Schématisation des valorisations réflexives.

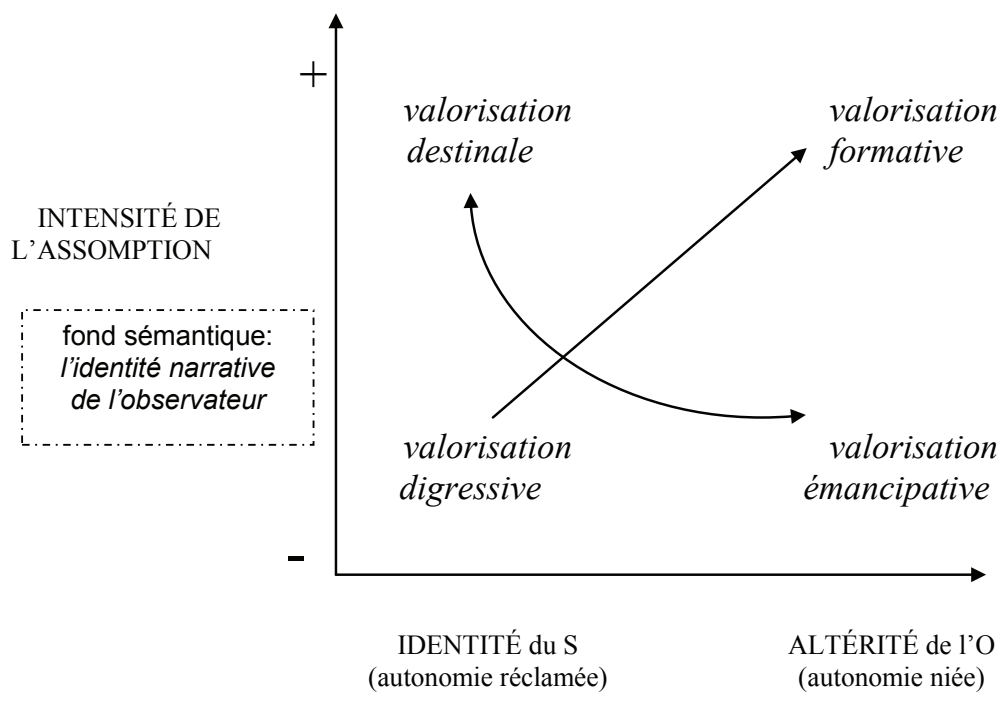

Pour ce qui concerne les valorisations transitives, nous gardons l'ancrage de la schématisation à la forme de vie de l'observateur (foyer de la valorisation) ; cela implique que l'identité de l'objet soit encore saisie comme l'altérité. On pourra distinguer les valorisations transitives suivantes :

e) les valorisations prothétiques indiquent que les valeurs identitaires de l'objet ne sont pas seulement assumées par le sujet observateur mais elles sont promues au rang d'éléments clés d'une restructuration identitaire : l'altérité de l'objet devient alors une prothèse, en niant son potentiel d'hétéronomie. On a déjà reconnu que les valeurs définissent bilatéralement des profils identitaires, en même temps que des relations interactantielles. Or, si la force gravitationnelle d'une identité prévaut totalement sur l'autre, on entre dans une actantialisation commune qui tend à suturer aussi les 
profils distingués dans la reformulation globale d'un seul corps actoriel ;

f) les valorisations instrumentales relèvent d'un servilisme dépourvu de toute dignité de médiation statutaire, c'est-à-dire que les valeurs identitaires de l'objet sont localement pliées à la préservation de certaines visées contingentes de l'observateur; l'hétéronomie de l'objet est presque totalement effacée et son rôle actantiel est inventé selon des exigences épisodiques ;

g) les valorisations ludiques sont concentrées sur des traits identitaires de l'objet et elles reconnaissent que ce dernier est une instance hétéronome ; cependant, elles tendent à manipuler les relations paradigmatiques et syntagmatiques de ces traits afin de démontrer la faible tenue de l'altérité et son incapacité à impliquer véritablement l'identité de l'observateur ;

h) les valorisations cultuelles tendent en revanche à absolutiser l'altérité de l'objet, au-delà du fait qu'elle soit capable de garantir effectivement des bénéfices au sujet observateur (en tant que foyer de la valorisation). La dévotion pour l'objet envisage une inversion des relations implicatives, de sorte que le sujet de la valorisation devient enfin un instrument pour la conservation et l'amplification de l'identité de l'objet. 
Figure 2 - Schématisation des valorisations transitives.

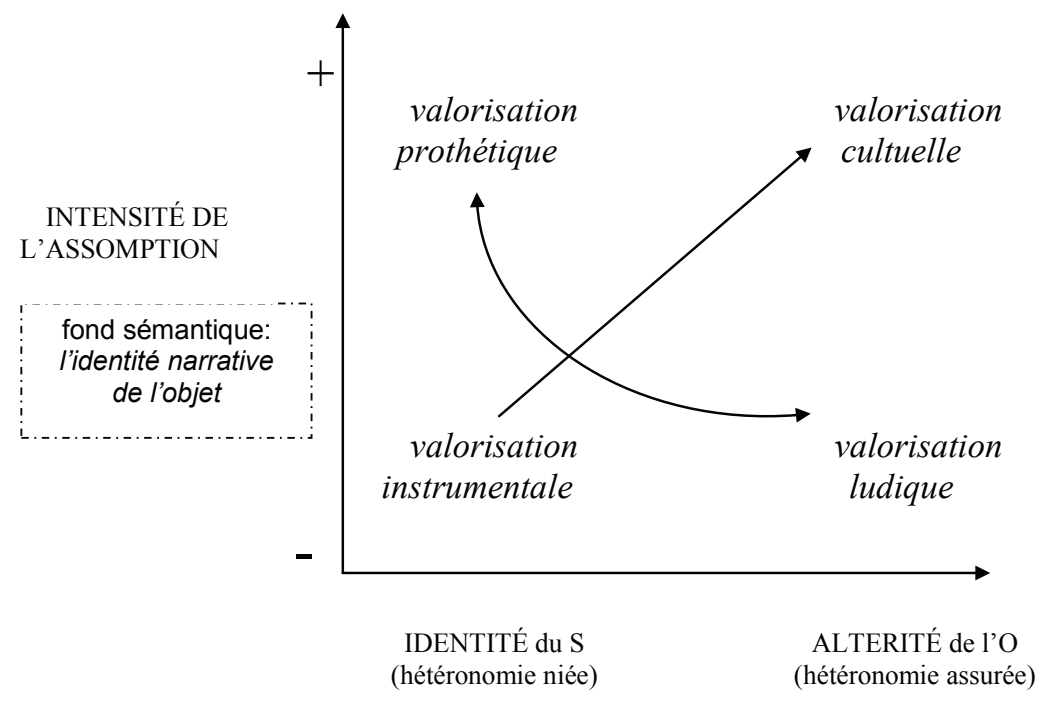

\section{Valorisations utopiques et Valeurs (avec une majuscule)}

On peut aussi dépasser les valeurs dépendantes d'une certaine scénarisation (la plus simple et la plus abstraite se limite à saisir la confrontation entre un sujet et un objet) et penser la prise d'initiative même comme une ré-implication capable de scénariser de nouveau toutes les relations. Cela explique l'utilisation du terme "valeur » pour désigner également des attitudes individuelles qui sont assumées paradoxalement comme l'état de santé d'une société : la générosité, le pardon, le courage, etc. Les «Valeurs » avec une majuscule sont des passions éthiques capables de requalifier des espaces institutionnalisés et régulés à cause des divergences d'intérêts et des prévarications (le droit contient en soi un caractère réparateur). Si la différence entre les valeurs d'usage et les valeurs de base relève encore d'une hiérarchisation fonctionnelle ou motivationnelle (elles sont 
tous les deux internes à la même configuration narrative) (DEWEY, 1929, p. 397), les Valeurs concerneraient un saut qualitatif, une refiguration ne permettant pas des calculs sur les relations futures. En effet, les premières (valeurs isotopiques) qualifient des parcours narratifs (directions de vie), souvent déjà envisagés par les praxis et les lois ; les secondes, en revanche, viseraient un cadre existentiel totalement restructuré, avec des implications extensives et sans des évaluations " économiques » préalables. Les " coûts » des Valeurs ne sont alors soutenus que par le support des croyances, étant donné que ce type de valeurs se nourrissent de valorisations utopiques. Le statut paradoxal des valorisations utopiques est lié à l'absence d'un espace où l'on puisse évaluer la consistance des relations actantielles actualisées par l'exercice des «Valeurs ». Par exemple, le pardon est l'acceptation d'un saut dans le vide, d'où le caractère risqué de l'« atterrage » dans un scénario nouveau, qui n'a plus mémoire des dettes sociales reconnues. La valence des «Valeurs » est alors l'absolutisation locale du déséquilibre (les Valeurs sont vertigineuses) aussi bien que la défocalisation des distances axiologiques entre partenaires de la communication; comme nous l'avons dit, le caractère unilatéral de l'exercice des «Valeurs » est retenu comme ressource fondamentale de la société, voire comme un indicateur de la santé d'une communauté entière : étant capables de réparer l'obsession de symétrisation du pacte social, même au risque d'incarner l'injustice subie jusqu'au bout, les héros de la société sont le plus souvent ses victimes.

L'utopie n'a pas de lieu assigné et le manque de consistance lui donne un statut provisoire ; toutefois, dans notre argumentation, l'utopie n'est pas la préfiguration d'un état idéal, mais le dépassement en amont de tous les 
" cadres de facilitation » (scénarisations prototypiques ou frames) normalement utilisés pour l'interprétation. Or, les valences organisées sous le régime des domaines sociaux ne peuvent pas satisfaire le besoin de valeurs locales capables de réinitialiser les confrontations et les implications : les Valeurs ont un valoir éthique parce qu'elles sont capables en premier lieu de croiser de nouveau des destins sociaux reconnus mais séparés. En effet, la société ne manque jamais d'autocommuniquer qu'elle n'est pas suffisante à ellemême. Les "Valeurs » sont ainsi la conscience trouble de la société, elles indiquent qu'une rationalisation absolue n'est pas souhaitable. Par exemple, il faut sortir certainement de la valeur d'échange, surtout si elle est impliquée dans des violations identitaires (œil pour œil, dent pour dent) ; c'està-dire qu'il faut sortir des résonances paradigmatiques et se poser au-delà de l'organisation garantie par les jeux de langage (à ce propos, selon Wittgenstein, l'éthique doit porter le langage jusqu'à ses limites constitutives).

Les « Valeurs » définiraient alors le rôle social réservé aux valorisations utopiques, lesquelles encourageraient les traductions entre les valeurs identitaires, voire les conversions axiologiques. Dans cette perspective, la « Valeur» avec une majuscule peut tout à fait correspondre à la définition générale de la valeur fournie au début de cet essai ; nous ne sommes pas devant un terme homonymique, car la Valeur exemplifie plutôt l'absolutisation implicative des signifiés quand ils ne sont pas protégés à l'intérieur d'une clôture de pertinence ou d'un programme narratif ; bref, elle exhibe le déséquilibre (la valence) sans échange, sans contrepartie certaine.

En ce qui concerne la valorisation utopique, elle implique une re-scénarisation des relations qui doit être compétitive par rapport aux scénarisations (I) institutionnelles (spécialisées 
selon les divers domaines), (II) transcendantes (enracinées dans un espace sacré inviolable), (III) régulatrices (élaborées par rationalité d'organisation). Les valorisations plus « traditionnelles » sont le plus souvent actualisées par défaut et selon une économie de confrontation capable d'établir des déséquilibres rentables, réflexifs ou transitifs ; en revanche, la valorisation utopique se fonde sur une dépense tout à fait personnelle qui exemplifie un « virement » imprévu et incertain du capital social acquis.

\section{L'engagement}

L'engagement semble être un bon exemple d'une valeur implicative institutionnalisée qui entre en concurrence avec sa version autonome, élective, "majuscule » : en effet, d'une part, l'engagement soutient un "gage », une dette, une promesse qui sollicite une direction de vie et une finalité ; de l'autre part, l'engagement se propose comme une implication infinitive qui symbolise une forme de vie entière (on pense à la figure « mythique » de l'intellectuel engagé). La dimension perfective de l'engagement est quantifiable et convertible dans une restitution finale sous le signe de la responsabilité limitée; la version imperfective de l'engagement est en revanche une implication illimitée, spécifique (elle affecte la totalité des attitudes individuelles). Les régimes d'engagement qualifient alors la gestion de valences et la négociation des formes d'implication. En particulier, l'engagement électif s'éprouve lui-même dans les conversions promues par l'exercice des Valeurs ; d'autres formes d'engagement se limitent à garantir aux Valeurs une hospitalité discursive et éventuellement à hospitaliser les pauvres diables qui les pratiquent avec un excès de conviction. La rhétorique des Valeurs est une 
transposition continue du moment de leur exercice, de sorte qu'à l'augmentation de leur nomination corresponde l'archéologie de leur dernière adoption. Il en va de même avec la proclamation de la mort des Valeurs : elle n'est que le rite apotropaïque qui nous libère de leur présence utopique.

\section{Évaluation et objectivation}

\section{Le geste évaluatif et l'objectivation : valeurs et faits}

Nous avons trop souligné peut-être le rôle des Valeurs dans l'engagement. Le geste évaluatif transforme plus généralement des liens implicatifs locaux en valeurs qui contraignent l'imputation parallèle de certaines valeurs identitaires de l'évaluateur. Le jugement de goût ne peut pas être réduit à des décisions interprétatives pour l'établissement d'un scénario de relations ; la valence qui informe les traits évalués est la même qui soutient les compétences de l'évaluateur. L'engagement implicite dans l'évaluation construit une symétrisation des valorisations transitives et réflexives : il y a donc un effet performatif, un geste justement. Le geste évaluatif se développe dans la visée d'un équilibre et il se propose même comme le paramètre d'une attitude critique $^{15}$; il exhibe l'existence de valences bilatéralement approfondies (goût du connaisseur/objet de bon goût) qui

15 «Any theory of values is perforce entrance into the field of criticism » (DEWEY, 1929, p. 398). Le point de contact entre le pragmatisme américain et la sémiotique est alors l'idée que les valeurs sont les fruits des évaluations récursives selon un enchaînement potentiellement infini des médiations. Du reste, la valeur immédiate, fixée par l'euphorie ou la dysphorie, est une illusion ; elle cache seulement sa compromission originaire avec des instances critiques qui habitent déjà la perception (la dissimilation entre des déformations virtuelles et réelles, la double dépendance du goût, etc.) et des valences qui expriment des médiations entre une situation expérientielle en acte et d'autres déjà vécues ou possibles. 
rendraient l'axiologie totalement intériorisée de l'expert plus fiable qu'une procédure d'objectivation (d'où la dévaluation de la méthode).

Par ailleurs, on ne peut pas sortir des médiations évaluatives car le choix même d'une approche scientifique est une préférence négociée ; mais on peut bien s'engager à expliciter de manière critique la recherche d'un équilibre entre les dépendances subjectales et objectales de la relation considérée. L'objectivation de la «non-neutralité » va de pair avec l'objectivité d'une implication qui oblige à définir sa position. À travers cette "duplicité », on a pu réclamer l'indistinction entre le fait et la valeur et dans le même temps échapper à un relativisme subjectiviste absolutisé ${ }^{16}$. Il n'y a pas de faits attestés sans une perspective de valorisation qui soutiendrait leur discrimination ; au demeurant, l'empirisme relève de sélections et d'emphatisations des contrastes.

Il n'y a pas d'objectivation sans méthode, de sorte que les « faits » de la science sont toujours réorganisables sous de nouvelles descriptions. À chaque méthode correspond une valence différente qui soutient la confiance des vérifications/ falsifications et la résolution de descriptions. Il y a donc une évaluation des méthodes aussi bien qu'une évaluation interne à l'exploitation de la méthode finalement choisie ; une évaluation pratiquée sur la base d'un intervalle de confiance par rapport à la portée et à l'échelle des échantillonnages et des mesures collectés. Cela ne compromet pas les valorisations transitives des relevés scientifiques sous une perspective d'objectivation ; plus simplement, on explicite les conditions pour argumenter la non-équivalence des méthodes et des relevés et la fiabilité de certaines modélisations, en sortant

16 Dewey (1988a, 1988b); Putnam (2004). 
d'un relativisme qui ne permet pas de choisir et d'apprécier des différents niveaux de consistance des descriptions. Bref, la valeur dans le domaine scientifique n'a aucun potentiel « destructeur »; elle ne compromet pas les résultats, mais elle leur assigne une valence pratique à l'intérieur d'un champ de choix épistémologiques.

\section{Saillances et prégnances : valeurs émergeantes et valeurs assumées}

Les saillances sont des points qualitatifs par rapport auxquels les valorisations transitives ou réflexives peuvent se partager le champ de la sémantisation. La base initiale de la division est la dialectique entre les scénarisations événementielles et pragmatiques qui motive la distribution des attributions de valeurs émergentes (saillances), mais bientôt l'observation de deuxième ordre permet une psychologisation de l'environnement et une "matérialisation critique » du milieu interne du sujet. Ce doublement transforme les enjeux des valorisations en un champ thématique (et problématique) de prégnances. D’abord, la prégnance n'est que le déséquilibre d'imputation de la force implicative, attribuée soit au milieu externe (physique, social, etc.), soit au milieu interne (biologique, psychique, etc.). Mais les prégnances déplacées dans l'environnement peuvent devenir une extension prothétique du sujet (extended mind) ; conjointement, l'événementialité interne (milieu psychologique) construit de nouvelles saillances qui attendent enfin des partages ultérieurs entre imputations (les convictions du soi et les perturbations du moi).

Réné Thom (1991) a remarqué que la propagation de prégnances procède par contiguïté (extension syntagmatique) ou par similarité (extension paradigmatique) de l'implication. 
Les prégnances dessinent une écologie de pertinentisation des saillances, mais leur propagation suggère aussi la recherche locale d'une saturation de la pertinence ; une recherche qui procède à travers la dynamisation du cadre relationnel afin de coaguler des fronts de valeurs opératives (prégnances transitives) et opérables (prégnances réflexives).

Selon Thom, la propagation des prégnances tendrait dans tous les cas à retourner vers les épicentres originaires de la diffusion ; l'implication extensive (par contiguïté ou par reflet paradigmatique) déclencherait un affaiblissement de l'intensité. La thématisation a donc des foyers d'ancrage qui resteraient des réserves d'intensité ; une fois devenus inaccessibles, ils provoqueraient la désémantisation de toute une région d'investissements existentiels. En effet, cette idée peut être aussi rebattue sur la dialectique entre valeurs perceptuelles et valeurs discursives, étant donné que le plan fictifdes opérations linguistiques organise une autonomisation d'évaluations qui toutefois maintiennent chroniquement la nécessité de se convertir dans desvalorisations expérientielles. Les émotions sont l'opérateur privilégié pour soustraire l'impression que l'espace discursif est un champ totalement stratégique, opérable sans scénarisation événementielle. L'asymétrie de la communication (énonciateur/énonciataire) est alors utilisée comme le moyen le plus simple de retrouver le «thrilling » des prégnances événementielles indépendantes de l'initiative stratégique du discours (l'interprète ne connaît pas d'avance les péripéties narratives ou les discontinuités discursives qui perturbent ses attentes). De manière plus générale, on cherche la conversion des simulacres discursifs dans des foyers de prégnances vécues, même dépourvues d'instance critique (la stimulation des émotions agencée par l'organisation discursive doit toujours se disculper de son 
chantage apparent).

Le monde de l'expérience perceptive est unique, c'està-dire qu'il oblige les prégnances à élire des saillances disponibles dans un espace vital d'ancrage ; en revanche, le discours se reproduit sous l'idée d'une multiplication des mondes de références où l'on pourra reformuler des individuations identitaires. La prégnance expérientielle est donc une sémantisation écologique qui s'empêche d'utiliser des valorisations qui mettent entre parenthèses les enjeux identitaires situés (ancrés dans un lieu de vie ) ; dans le discours, en revanche, l'économie des valorisations se dédouble dans des rôles d'expression et des rôles de contenu, et les saillances ne sont que les traits qui s'offrent à chaque fois comme les pivots de nouvelles pertinentisations, en profitant ultérieurement d'un terrain discursif mis en place (on pense par exemple aux passages entre les sémantisations figuratives, plastiques et enfin figurales, qui caractérisent la pleine jouissance d'un texte artistique).

\section{La coagulation}

La détermination de formes est toujours dépendante d'une valorisation et n'est donc jamais dépourvue d'enjeux sémantiques ; toutefois, la valeur est à son tour dépendante d'un diagramme de relations actancielles, donc d'une forme. L'établissement d'un primat de la forme sur la valeur ou viceversa est trompeuse : l'implication est le seul moyen de se positionner dans une scène, et « trouver » la manière de s'impliquer n'est que l'acceptation d'une coagulation de déterminations bilatérales. Il faut " faire prise », même au risque de subir des déterminations exogènes : la forme relationnelle ne précède ni ne suit, mais coagule. 
Enfin, la coagulation n'est que l'établissement des frontières entre instances motivées (ou déléguées) à se définir réciproquement; une fois définies, les instances peuvent bien chercher une fluidification des rapports et des moments plus informels. C'est le risque d'un vertige identitaire qui motive rythmiquement des exigences de confrontations et de prises, même sous la forme d'un reflux d'agressivité.

$\mathrm{Au}$ demeurant, l'orientation aux valeurs contraint à « aller au front » pour chercher une distinction. On peut expliquer ainsi la conception de la valeur dans les stratégies de marketing ; dans ce domaine, les valeurs ne sont que des agrégats de sens testés dans des fronts de différentiations, c'est-à-dire qu'elles sont des ressources de distinction.

\section{Les valeurs figuratives}

\section{Paradoxe du bagage actoriel}

Il faut créer en avance de la valeur pour être vraiment compétitif, il faut anticiper le " front » et se garantir un équipement qui puisse dessiner les «batailles » à son profit. Le stock des valeurs élaborées n'est alors qu'un bassin de traits actoriels, un équipement valable qui peut rétablir ou reconfigurer un espace d'entreprise, d'investissement de soi. On trouve ici le paradoxe de la valeur, définie « au front » selon une sorte de cocréation avec les interactants, qui se traduit dans un bagage actoriel construit par l'exercice, mais qui fait également appel à une réserve de traits thésaurisés dans la densité figurative de sa propre constitution. Ce paradoxe explore la compatibilité entre la nécessité des valeurs d'être en acte et leur présence nichée dans une déclinaison figurative surabondante. La forme de vie des acteurs sociaux n'est que 
la résolution de ces besoins antithétiques ; cette résolution constitue des pôles identitaires en dormance, aussi bien que des fronts ouverts de définitions de soi en osmose constante avec d'autres formes de vie.

Dans cette perspective, la connaissance même pourrait apparaître comme la germination souterraine des valeurs associée à la mémoire des valences qui ne sont plus en acte (les choses continuent à vivre en nous-mêmes selon des élaborations mnésiques ou imaginatives). La connaissance est donc le symptôme le plus évident que la vie prend ses réserves par rapport à l'investissement direct, en trouvant par compensation un équilibre dans l'augmentation des possibilités identitaires. Expérience et connaissance deviennent deux régimes de convocation et d'élaboration de valeurs.

La consommation des valeurs se produit normalement dans leur exploitation actantielle, laquelle peut désémantiser la réserve actorielle ; d'ailleurs, la pure accumulation des valeurs « dormantes » se transformerait facilement en quantification laconique des capitaux à valence progressivement évanescente. Pour cette raison, on cherche l'échange afin de laisser les valeurs désémantisées pour d'autres investissements, même s'ils sont valables seulement en raison de leur nouveauté. Le marché identitaire oblige à une cotation continue (estimation des valences préservées) et à des permutations/conversions faites au bon moment. Or, la gestion rhétorique des valeurs assigne normalement à la quantification la tâche de construire le support argumentatif le plus approprié pour se promouvoir comme «valable pour tous ». En revanche, l'incommensurabilité des valeurs semble être résolue sur le plan argumentatif à travers des métaphores ou exaltée à travers l'unicité qualitative (MEYER, 2008, p. 142). 


\section{Consistance figurative et incomplétude actorielle}

On pourrait enfin attribuer à la dialectique entre quantification et appréciation qualitative (BORDRON, 2011) la capacité de distinguer les types de valences : les valences modales, qui garantiraient une consistance narrative aux scénarisations, et les valences constitutionnelles, qui contribueraient à la richesse figurative des confrontations actancielles. Si la consistance narrative permet d'attribuer des rôles actanciels aux acteurs par rapport à une économie des manques, la richesse figurative ouvrirait d'autres débauchés actantiels par rapport à une densité de traits prometteurs, même si partiellement « dormants ». L'exhibition des valeurs permet de modaliser immédiatement les relations selon une confrontation asymétrique des attributs identitaires; en revanche, la réserve (ou la retenue) des valeurs empêche la soumission aux scènes pratiques préorganisées et souvent provocatrices. D'ailleurs, la réalisation des valeurs visées n'est que l'avant-dernière réponse à une incomplétude actorielle ou à une surabondance inexprimée ; c'est donc une réalisation paradoxale, avec un côté positif (reconfiguration actorielle) et un côté négatif (elle démontre d'autres incomplétudes, d'autres manques).

La valeur exprime un «valoir » qui est à la fois constitutif (économie figurative intra-identitaire) et narrative (transformation de relations interidentitaires et interpénétrations des formes de vie) (BASSO FOSSALI, 2012). La reconnaissance de la valeur établit le remplissage d'un vide (coagulation implicative) et, dans le même temps, densifie d'autres possibilités de détermination identitaire. La plurivalence de la valeur lui permet de jouer un rôle crucial dans les opérations de conversion entre les scénarisations ; 
en effet, on parle de "valeur figurative » sans pléonasme pour souligner son détachement local de l'élaboration narrative afin d'entrer dans des raisonnements figuraux. La valeur devient alors un levier pour perfectionner à outrance un projet identitaire aussi bien que pour faire exploser une configuration stabilisée.

\section{Bilan partiel d'une petite enquête sur la valeur}

\section{Pour une écologie des valeurs}

Le traitement récursif et infatigable de la valeur se présente paradoxalement comme l'inacceptation chronique d'une implication déjà destinée aux individuations bilatérales des identités ; on neutralise ou on transforme leur potentiel d'implications (transpositions discursives), on réduit l'hétérogénéité de leur valence à travers la quantification, on souligne l'existence des valeurs qui dépassent les cadres de facilitation utilisés normalement pour leur socialisation (Valeurs avec une majuscule), etc. Du fait, les valeurs assignées sauvegardent en négatif les valeurs choisies, tout comme les valeurs uniques rendent souhaitables les valeurs redondantes ou itératives. Les équilibres et les déséquilibres des valeurs engendrent des valences réflexives (auto-attribution) et des valences transitives (imputation), infléchies par des régimes de coagulation et des régimes de fluidification qui donnent un rythme à la régénération des enjeux, en promouvant tantôt des moments de confrontation polémique ou contractuelle, tantôt des moments d'enrichissement et redéploiement figuratif. Toute une écologie des valeurs semble émerger.

Pour cette raison, la sémiotique pourrait développer autour du concept de valeur une perspective écosystémique 
qui semble être un bon viatique pour se repositionner à l'intérieur des sciences de la culture : en particulier, on pourrait conduire des enquêtes critiques sur les « coûts » des valeurs dans une forme de vie spécifique, par rapport à la consistance des pôles identitaires aussi bien que par rapport à la fluidité des rapports osmotiques avec d'autres formes de vie. Dans cette perspective on comprendrait peut-être mieux les équilibres de dépense des valeurs et la cocréation de valeurs nouvelles dans des fronts communs, étant donné que les formes de vie sont interpénétrées.

Une définition de la valeur en tant que lien implicatif ne saurait qu'étendre et généraliser le rôle spécifique des axiologies dans le partage des relations signifiantes communes. Par exemple, le détachement critique par rapport aux axiologies dominantes peut aussi naître comme une nécessité écologique d'éviter l'absolutisation vertigineuse des ressorts modaux institutionnalisées. D'ailleurs, la polémologie sociale fonctionne comme une série de miroirs qui montrent dans l'adversaire réfléchi la formule éventuelle de l'implication niée, voire de l'émancipation.

\section{Les facettes de la valeur}

La gestion des valeurs soumet toutes les analogisations (extractions diagrammatiques et projections figurales) et les prédications (infléchies par les modes d'existence) aux assomptions identitaires. Il s'ensuit que les valeurs, même si elles sont définies par des relations, ont comme déséquilibre local une détermination identitaire, et en particulier une imputation ou une auto-attribution des traits. En outre, comme nous l'avons vu, les valeurs développent une manifestation surabondante qui devient la réserve 
pour des actantialisations ultérieures. La thématisation actorielle répond à une élaboration des fronts identitaires et à une préorganisation narrative qui engendre toutefois une surabondance des possibilités. En particulier, la thématisation actorielle se concentre sur les pièges de la figurativité, sur les détails et les espaces de l'intimité, en problématisant la reconnaissance réciproque des sujets.

La valeur est au cœur de la sémiotique et, pour cette raison, on pourrait regretter qu'enfin sa conceptualisation soit trop facettée, voire hétéroclite. Toutefois, elle incarne bien le paradoxe interne à la gestion du sens, qui d'une part cherche la totalisation des attributions (chaque valeur recouvre alors un rôle différentiel) et de l'autre vise à dépasser - même utopiquement - les limites de toutes les configurations subies et contraignantes (les rôles sont alors une réouverture vertigineuse ou cathartique).

Pour synthétiser, nous pouvons reconnaître quatre aspects constitutifs de la valeur :

(i) l'insistance (être différence) : c'est la valeur en tant que relation qui rend possible des différends identitaires sur la base d'implications communes et partageables (assimilation/ dissimilation). La valeur est alors l'enracinement du sens à l'intérieur du couplage entre un épicentre de valorisation et un espace de référence en cohabitation (ego/alter). En particulier, l'insistance ne cesse jamais de chercher des déterminations actorielles ultérieures, en thésaurisant une richesse figurative qui dépasse les sollicitations actantielles en acte dans les procès décisionnels ;

(ii) la valence (faire la différence) : c'est le déséquilibre de chaque expérience du sens, par exemple la valence modale 
qui caractérise un trait figuratif par rapportà une configuration visée, soit fermée soit ouverte. Plus généralement, la valence est l'appréciation qualitative et distinctive d'une implication assignée (ou auto-attribuée) en comparaison d'autres valeurs en acte. Par rapport à l'insistance, qui a plutôt une attitude extensive, la valence tend à s'appliquer récursivement (questionnement du valoir) et à chercher un progrès d'intensité et de résolution interprétative ; toutefois, elle peut se résoudre aussi dans une simple sélection, par exemple quand on choisit des valeurs en fonction de leur inhérence sur le plan expérientiel ;

(iii) l'équipollence (mesurer la différence) : elle exprime la substituabilité de la valeur, son homologation comparative avec d'autres valeurs. L'équipollence indique aussi une familiarité avec cette valeur, une «fréquentation » qui a permis aussi l'élaboration de paramètres mesurant son « valoir ». La cotation de la valeur fait de la fluctuation de son valoir une dynamique d'offres d'échange, toutes indifférentes aux liens passés ; on profite alors de la désémantisation en tant qu'effet collatéral de la familiarisation pour chercher la réapparition de valences (prépondérances ${ }^{17}$ ) tout au long $\mathrm{du}$ processus d'échange d'une valeur avec l'autre (c'est une obsession qui incite typiquement les consommations immodérées);

(iv) la transcendance (transposer la différence) : elle expliquecommentl'insistance delavaleurpeutsechargerd'une valence émancipatrice qui scénarise de nouveau les relations, même au risque de devenir vertigineuse. La transcendance de

17 Dans notre excursus, les traitements combinés des valeurs qui donnent lieu aux choix n'ont, pour des raisons d'espace, pas reçu l'attention nécessaire (BERTHOZ, 2003, p. 37). 
la valeur met en jeu une instance critique qui pousse le sujet à dépasser ses intérêts et à se vouer à une cause hétéronome. La transcendance ne manifeste que l'attitude la plus propre à la culture, c'est-à-dire s'émanciper des liens sociaux déjà stipulés pour en chercher de nouveaux. Mais elle signale aussi la tentative d'aller au-delà de l'implication personnelle, selon une attitude éthique (Valeurs avec une majuscule). Toutefois, il faut remarquer que la transcendance de la valeur est souvent considérée comme le simple dépassement des visées strictement performatives et économiques ; par exemple, les marques mettent en avant des valeurs comme l'harmonie, l'amitié, la spontanéité pour se promouvoir comme des logiciels identitaires qui dépassent les raisons commerciales et qui peuvent s'étendre aussi à l'investissement direct de la forme de vie de l'acteur social, en conférant la sensation d'une transcendance par rapport aux valeurs d'échange et d'usage. Le risque naturellement est que ces «valeurs » finissent par rééditer des idéaux partageables (politiquement corrects) d'une humanité toujours plus fictive et par devenir enfin des «passions désubjectivisées » (MEYER, 2008, p. 195) et hypocrites, voire des principes régulateurs pour ennoblir les consommations de notre sémiosphère même.

\section{RÉFÉRENCES}

BASSO FOSSALI, P. Possibilisation, disproportion, interpénétration : trois perspectives pour enquêter sur la productivité de la notion de forme de vie en sémiotique. Nouveaux Actes Sémiotiques, n. 115, 2012. Disponible sur: <http://revues.unilim.fr/nas/document.php?id=4138>. Accédé le : 25 sept. 2012.

. Peirce et la photographie. In: BASSO FOSSALI , P.; 
DONDERO, M. G. Sémiotique de la photographie. Limoges: PULIM, 2011.

- Création et restructuration identitaire. Pour une sémiotique de la créativité. RS/SI, Arts du faire, v. 27, n. 1, 2009a.

. La tenuta del senso. Per una semiotica della percezione. Roma: Aracne, 2009b.

. La promozione dei valori. Semiotica della comunicazione e dei consumi. Milano: Franco Angeli, 2008a. . Vissuti di significazione. Pisa: ETS, 2008b.

. Semiotica dello spazio e semantica storica del giardino: esplorazione di metodo e appunti per una ricerca. Visible, Limoges, PULIM, n. 2, 2006.

BERTHOZ, A. La décision. Paris : Odile Jacob, 2003.

BORDRON, J.-F. Trois ordres de la valeur selon la qualité, la quantité et la relation. Semen, n. 32, 2011.

CLAM, J. Sciences du sens. Perspectives théoriques. Strasbourg : Presses Universitaires de Strasbourg, 2006.

DEWEY, J. Existence and Value. In: Existence and Value. London: George Allen, 1929. p. 394-437.

. Theory of Valutation [1939]. In: . The Later Works of John Dewey. v. 13. Carbondale: Southern Illinois University Press, 1988a.

. Some Questions about Value [1944]. In: . The

Later Works of John Dewey. v. 15. Carbondale: Southern Illinois University Press, 1988b.

FONTANILLE, J. Pratiques sémiotiques. Paris, PUF, 2008.

GREIMAS, A. J. De l'imperfection. Périgueux: Fanlac, 1986. 
MEYER, M. Principia Rhétorica. Une théorie générale de l'argumentation. Paris: Fayard, 2008.

PETITOT, J. Les deux indicibles, ou la sémiotique face à l'imaginaire comme chair. In : PARRET, H. ; RUPRECHT, H.G. (Org.). Exigences et perspectives de la sémiotique. v. 1. Amsterdam: John Benjamins, 1985. p. 283-305.

PUTNAM, H. Fait/Valeur : la fin d'un dogme et autres essais. Paris: Éditions de l'Éclat, 2004.

RASTIER, F. Deniers et Veau d'or : des fétiches à l'idole. Texto! (en ligne), 2004. Disponible sur: <http://www.revue-texto. net/Inedits/Rastier/Rastier_Deniers.html>. Accédé le : 01 sept. 2014.

La mesure et le grain. Sémantique de corpus. Paris : Honoré Champion, 2011.

Arts et sciences du texte. Paris: PUF, 2001.

SAUSSURE, F. de. Écrits de linguistique générale. Paris: Gallimard, 2002.

THOM, R. Apologie du Logos. Paris: Hachette, 1991.

Artigo recebido em outubro de 2014 e aprovado em novembro de 2014.

Disponível em: http://seer.fclar.unesp.br/casa 